\title{
Exact Discrete Analogs of Derivatives of Integer Orders: Differences as Infinite Series
}

\author{
Vasily E. Tarasov \\ Skobeltsyn Institute of Nuclear Physics, Lomonosov Moscow State University, Moscow 119991, Russia \\ Correspondence should be addressed to Vasily E. Tarasov; tarasov@theory.sinp.msu.ru
}

Received 30 July 2015; Accepted 8 October 2015

Academic Editor: Mario Ohlberger

Copyright (C) 2015 Vasily E. Tarasov. This is an open access article distributed under the Creative Commons Attribution License, which permits unrestricted use, distribution, and reproduction in any medium, provided the original work is properly cited.

New differences of integer orders, which are connected with derivatives of integer orders not approximately, are proposed. These differences are represented by infinite series. A characteristic property of the suggested differences is that its Fourier series transforms have a power-law form. We demonstrate that the proposed differences of integer orders $n$ are directly connected with the derivatives $\partial^{n} / \partial x^{n}$. In contrast to the usual finite differences of integer orders, the suggested differences give the usual derivatives without approximation.

\section{Introduction}

It is well known that the finite difference of integer order $n$ cannot be considered as an exact discretization of the derivative of order $n$. For example, the forward finite difference $\Delta_{h}^{1}$ of first order with step $h$ can be formally represented $[1,2]$ by the Taylor series:

$$
\Delta_{h}^{1}=h \frac{\partial}{\partial x}+\frac{h^{2}}{2} \frac{\partial^{2}}{\partial x^{2}}+\frac{h^{3}}{6} \frac{\partial^{3}}{\partial x^{3}}+\cdots=\exp \left(h \frac{\partial}{\partial x}\right)-I
$$

Therefore the finite differences of order $n \in \mathbb{N}$ give only approximation of the derivatives of integer orders $\partial^{n} / \partial x^{n}$, and $\Delta_{h}^{n} / h^{n}$ gives the derivative at $h \rightarrow 0$ only. Using formal inverting of (1), we get the equation

$$
h \frac{\partial}{\partial x}=\log \left(1+\Delta_{h}^{1}\right)=\sum_{j=0}^{\infty} \frac{(-1)^{j}}{j+1} \Delta_{h}^{j+1},
$$

which holds in the sense that left and right sides of (2) give the same result when applied to polynomials and analytic functions. Equation (2) allows us to assume that the derivatives of integer orders should be represented by differences that are represented by infinite series with power-law coefficients. For this reason, these differences will be called the infinite but they are not supposed infinite in value.

It should be noted that infinite differences and corresponding derivatives of noninteger order have been first proposed by Grünwald [3] in 1867 and independently by
Letnikov [4] in 1868. These differences are defined by infinite series (see Section 20 in [5]) as a generalization of the usual finite difference of integer orders. Now these differences are called the Grünwald-Letnikov fractional differences $[5,6]$.

In this paper, we suggest new differences of integer orders that are connected with derivatives not approximately. A characteristic property of the suggested differences is that its Fourier series transforms have the power-law form. We demonstrate that these differences of integer orders $n$ are directly connected with the derivatives $\partial^{n} / \partial x^{n}$. The main advantage of these differences is a direct connection with the usual derivatives for all integer orders.

\section{Derivatives and Differences of Integer Orders}

In this section we briefly describe the finite differences and derivatives of integer orders to fix notation for further consideration.

2.1. Finite Differences and Derivatives. Let us consider wellknown finite differences of integer orders $n \in \mathbb{N}$.

Definition 1. The forward finite difference $\Delta_{h}^{n}$ of order $n \in \mathbb{N}$ with the step $h$ is given by

$$
\Delta_{h}^{n} f(x):=\sum_{m=0}^{n} \frac{(-1)^{m} n !}{m !(n-m) !} f(x+(n-m) h) .
$$


The backward finite difference $\nabla_{h}^{n}$ of order $n \in \mathbb{N}$ is given by

$$
\nabla_{h}^{n} f(x):=\sum_{m=0}^{n} \frac{(-1)^{m} n !}{m !(n-m) !} f(x-m h) .
$$

The central finite difference $\delta_{h}^{n}$ of order $m \in \mathbb{N}$ is given by

$$
\delta_{h}^{n} f(x):=\sum_{m=0}^{n} \frac{(-1)^{m} n !}{m !(n-m) !} f\left(x+\left(\frac{n}{2}-m\right) h\right)
$$

These finite differences of order $m \in \mathbb{N}$ for functions $f[n]$ of a discrete variable $n \in \mathbb{Z}$ are defined by the equations

$$
\begin{aligned}
& \Delta^{m} f[n]:=\sum_{j=0}^{m} \frac{(-1)^{j} m !}{j !(m-j) !} f[n+(m-j)], \\
& \nabla^{m} f[n]:=\sum_{j=0}^{m} \frac{(-1)^{j} m !}{j !(m-j) !} f[n-j], \\
& \delta^{m} f[n]:=\sum_{j=0}^{m} \frac{(-1)^{j} m !}{j !(m-j) !} f\left[n+\left(\frac{m}{2}-j\right)\right] .
\end{aligned}
$$

Let us give a connection of the finite differences and derivatives of integer orders.

Proposition 2. The finite differences (3)-(5) of order $n \in \mathbb{N}$ with the step $h$ can be represented by the equations

$$
\begin{aligned}
& \Delta_{h}^{n} f(x)=\sum_{m=0}^{n} \frac{(-1)^{m} n !}{m !(n-m) !} \exp \left((n-m) h \frac{\partial}{\partial x}\right) f(x), \\
& \nabla_{h}^{n} f(x)=\sum_{m=0}^{n} \frac{(-1)^{m} n !}{m !(n-m) !} \exp \left(-m h \frac{\partial}{\partial x}\right) f(x), \\
& \delta_{h}^{n} f(x) \\
& \quad=\sum_{m=0}^{n} \frac{(-1)^{m} n !}{m !(n-m) !} \exp \left(\left(\frac{n}{2}-m\right) h \frac{\partial}{\partial x}\right) f(x) .
\end{aligned}
$$

Proof. Using the well-known relation [7] of the form

$$
\exp \left(h \frac{\partial}{\partial x}\right) f(x)=f(x+h)
$$

we get

$$
\begin{aligned}
f(x+(n-m) h) & =\exp \left((n-m) h \frac{\partial}{\partial x}\right) f(x), \\
f(x-m h) & =\exp \left(-m h \frac{\partial}{\partial x}\right) f(x), \\
f\left(x+\left(\frac{n}{2}-m\right) h\right) & =\exp \left(\left(\frac{n}{2}-m\right) h \frac{\partial}{\partial x}\right) f(x) .
\end{aligned}
$$

Substituting (11) into the finite differences (3)-(5) give representation (9).
Remark 3. For example, the central finite difference of second order is represented [7] in the form

$$
\delta_{h}^{2}=2 \sum_{j=1}^{\infty} h^{2 j} \frac{1}{(2 j) !} \frac{\partial^{2 j}}{\partial x^{2 j}} .
$$

It is easy to see that

$$
\frac{1}{h^{2}} \delta_{h}^{2} f(x) \neq \frac{\partial^{2} f(x)}{\partial x^{2}} .
$$

Only the limit $h \rightarrow 0$ gives

$$
\lim _{h \rightarrow 0} \frac{\delta_{h}^{2} f(x)}{h^{2}}=\frac{\partial^{2} f(x)}{\partial x^{2}} .
$$

As a result, we have that the central finite difference $\delta_{h}^{2}$ can be considered only as an approximation of the derivative of second order. Similarly, we have the inequality

$$
\frac{1}{h^{n}} \delta_{h}^{n} f(x) \neq \frac{\partial^{n} f(x)}{\partial x^{n}} \quad(n \in \mathbb{N}),
$$

and the equality for the limit $h \rightarrow 0$ :

$$
\lim _{h \rightarrow 0} \frac{\delta_{h}^{n} f(x)}{h^{n}}=\frac{\partial^{n} f(x)}{\partial x^{n}} .
$$

For other finite differences (3)-(4) we have equations, which are similar to (15) and (16).

Using representations (9) and (15), we can see that all finite differences (3)-(5) of orders $n \in \mathbb{N}$ cannot give exactly the derivative $\partial^{n} / \partial x^{n}$. These differences can be considered only as approximation of the derivative of integer order $n$ which coincide with derivatives at $h \rightarrow 0$ only.

2.2. Fourier Series Transform and Finite Differences. A conclusion about connection between finite differences and derivatives can also be obtained by using the Fourier series and integral transforms of differences (6)-(8) and derivatives.

The Fourier integral transform of the function $f(x)$ is

$$
\widetilde{f}(k)=\int_{-\infty}^{+\infty} d x f(x) e^{-i k x}=\mathscr{F}\{f(x)\},
$$

and the inverse Fourier integral transform is defined by

$$
f(x)=\frac{1}{2 \pi} \int_{-\infty}^{+\infty} d k \tilde{f}(k) e^{i k x}=\mathscr{F}^{-1}\{\tilde{f}(k)\} .
$$

It is well known that the derivatives of integer order $n \in \mathbb{N}$ have the Fourier integral transform in the form

$$
\begin{aligned}
\mathscr{F}\left(\frac{\partial^{n} f(x)}{\partial x^{n}}\right) & =\int_{-\infty}^{+\infty} d x e^{-i k x}\left(\frac{\partial^{n} f(x)}{\partial x^{n}}\right) \\
& =(i k)^{n} \tilde{f}(k) .
\end{aligned}
$$

The Fourier series transform of the discrete function $f[n] \in l^{2}$ is defined by

$$
\widehat{f}(k)=\sum_{n=-\infty}^{+\infty} f[n] e^{-i k n h}=\mathscr{F}_{h, \Delta}\{f[n]\} .
$$


And the inverse Fourier series transform is

$$
f[n]=\frac{h}{2 \pi} \int_{-\pi / h}^{+\pi / h} d k \widehat{f}(k) e^{i k n h}=\mathscr{F}_{h, \Delta}^{-1}\{\widehat{f}(k)\} .
$$

Note that we use the minus sign in the exponent of (20) instead of plus that is usually used.

The Fourier series transforms of the finite differences of order $n \in \mathbb{N}$ are given by the following proposition.

Proposition 4. The Fourier series transforms of differences (6)-(8) have the form

$$
\begin{aligned}
& \mathscr{F}_{h, \Delta}\left(\Delta^{m} f[n]\right) \\
& =\sum_{j=0}^{m} \frac{(-1)^{j} m !}{j !(m-j) !} \exp (i k(m-j) h) \widehat{f}(k), \\
& \mathscr{F}_{h, \Delta}\left(\nabla^{m} f[n]\right)=\sum_{j=0}^{m} \frac{(-1)^{j} m !}{j !(m-j) !} \exp (-i k j h) \widehat{f}(k), \\
& \mathscr{F}_{h, \Delta}\left(\delta^{m} f[n]\right) \\
& =\sum_{j=0}^{m} \frac{(-1)^{j} m !}{j !(m-j) !} \exp \left(i k\left(\frac{m}{2}-j\right) h\right) \widehat{f}(k) .
\end{aligned}
$$

Proof. Let us consider the forward finite difference (6). Using (20), we get

$$
\begin{aligned}
\mathscr{F}_{h, \Delta} & \left(\Delta^{m} f[n]\right) \\
= & \sum_{j=0}^{m} \frac{(-1)^{j} m !}{j !(m-j) !} \sum_{n=-\infty}^{+\infty} e^{-i k n h} f[n+(m-j)] \\
= & \sum_{j=0}^{m} \frac{(-1)^{j} m !}{j !(m-j) !} \exp (i k(m-j) h) \\
\cdot & \sum_{n^{\prime}=-\infty}^{+\infty} e^{-i k n^{\prime} h} f\left[n^{\prime}\right] \\
= & \sum_{j=0}^{m} \frac{(-1)^{j} m !}{j !(m-j) !} \exp (i k(m-j) h) \widehat{f}(k) .
\end{aligned}
$$

As a result, we proved (22). Similarly, we get (23) and (24).

Remark 5. It is easy to see that the Fourier series transform of differences (6)-(8) of order $n \in \mathbb{N}$ cannot be considered as $n$ power of $(i h k)$ :

$$
\mathscr{F}_{h, \Delta}\left(\Delta^{n}\right) \neq(i k h)^{n} .
$$

Only the limit $h \rightarrow 0$ gives

$$
\lim _{h \rightarrow 0} \frac{\mathscr{F}_{h, \Delta}\left(\Delta^{n}\right)}{h^{n}}=(i k)^{n} .
$$

For other finite differences (4)-(5), we have equations, which are similar to (26) and (27).
Proposition 6. The inverse Fourier integral transform (18) of expressions (22)-(24) has the form

$$
\begin{aligned}
& \mathscr{F}^{-1}\left(\mathscr{F}_{h, \Delta}\left(\Delta^{m} f[n]\right)\right) \\
& =\sum_{j=0}^{m} \frac{(-1)^{j} m !}{j !(m-j) !} \exp \left((m-j) h \frac{\partial}{\partial x}\right) f(x), \\
& \mathscr{F}^{-1}\left(\mathscr{F}_{h, \Delta}\left(\nabla^{m} f[n]\right)\right) \\
& =\sum_{j=0}^{m} \frac{(-1)^{j} m !}{j !(m-j) !} \exp \left(-j h \frac{\partial}{\partial x}\right) f(x), \\
& \mathscr{F}^{-1}\left(\mathscr{F}_{h, \Delta}\left(\delta^{m} f[n]\right)\right) \\
& =\sum_{j=0}^{m} \frac{(-1)^{j} m !}{j !(m-j) !} \exp \left(\left(\frac{m}{2}-j\right) h \frac{\partial}{\partial x}\right) \widehat{f}(k) .
\end{aligned}
$$

Proof. Let us consider (22) for the forward difference (6). Using the inverse Fourier integral transform (18) for the function $\exp (i k(m-j) h)$, we get

$$
\begin{aligned}
\mathscr{F}^{-1} & (\exp (i k(m-j) h)) \\
= & \frac{1}{2 \pi} \int_{-\infty}^{+\infty} d k \exp (i k(m-j) h) e^{i k x} \\
& =\frac{1}{2 \pi} \sum_{s=0}^{\infty} \frac{((m-j) h)^{s}}{s !} \int_{-\infty}^{+\infty} d k(i k)^{s} e^{i k x} \\
& =\sum_{s=0}^{\infty} \frac{((m-j) h)^{s}}{s !} \frac{\partial^{s}}{\partial x^{s}}=\exp \left((m-j) h \frac{\partial}{\partial x}\right) .
\end{aligned}
$$

As a result, we proved (28). Similarly, we get (29) and (30).

Remark 7. It is easy to see that expressions (22)-(24) coincide with (11) by the change of variables $j \rightarrow m$ and $m \rightarrow n$.

Remark 8. For example, the central finite difference (8) of second order has the Fourier series transform in the form

$$
\mathscr{F}_{h, \Delta}\left\{\delta^{2}\right\}=2 \sum_{j=1}^{\infty} \frac{(-1)^{j}}{(2 j) !}(k h)^{2 j} .
$$

Using (19), the inverse Fourier integral transform $\mathscr{F}^{-1}$ gives

$$
\mathscr{F}^{-1}\left\{\mathscr{F}_{h, \Delta}\left\{\delta^{2}\right\}\right\}=2 \sum_{j=1}^{\infty} \frac{1}{(2 j) !} h^{2 j} \frac{\partial^{2 j}}{\partial x^{2 j}},
$$

which coincides with (12).

As a result, we have that the characteristic property of finite difference (6) is the inequality

$$
\mathscr{F}_{h, \Delta}\left(\Delta^{n}\right) \neq(i k h)^{n} .
$$

This inequality leads us to the corresponding inequality

$$
\frac{1}{h^{n}} \mathscr{F}^{-1}\left(\mathscr{F}_{h, \Delta}\left(\Delta^{n}\right)\right) \neq \frac{\partial^{n}}{\partial x^{n}},
$$


which means that finite difference (6) of orders $n \in \mathbb{N}$ cannot give exactly the derivative $\partial^{n} / \partial x^{n}$. Only in the limit $h \rightarrow 0$, we get

$$
\lim _{h \rightarrow 0} \frac{\mathscr{F}^{-1}\left(\mathscr{F}_{h, \Delta}\left(\Delta^{n}\right)\right)}{h^{n}}=\frac{\partial^{n}}{\partial x^{n}} .
$$

Therefore difference (6) can be considered only as approximation of the derivative $\partial^{n} / \partial x^{n}$. For the finite differences $\nabla^{n}$ and $\delta^{n}$, which are defined by (7) and (8), we have the same equations.

\section{Formulation of the Problem}

Finite differences of orders $n \in \mathbb{N}$ can be considered only as approximation of the derivative of integer order $n$. We would like to get a difference that can be connected with the derivative $\partial^{n} / \partial x^{n}$ not only by the limit $h \rightarrow 0$.

In order that a difference of order $n \in \mathbb{N}$, which will be denoted by ${ }^{\mathcal{T}} \Delta^{n}$, corresponds to the derivative $\partial^{n} / \partial x^{n}$ not approximately, this difference should satisfy the condition

$$
\frac{1}{h^{n}} \mathscr{F}^{-1}\left(\mathscr{F}_{h, \Delta}\left({ }^{\mathscr{T}} \Delta^{n}\right)\right)=\frac{\partial^{n}}{\partial x^{n}} .
$$

This condition can be realized if this difference has the Fourier series transform in the form

$$
\mathscr{F}_{h, \Delta}\left({ }^{\mathscr{T}} \Delta^{n}\right)=(i k h)^{n} .
$$

Using (38), we can get an exact expression of the required difference $^{\mathscr{T}} \Delta^{n}$.

Let us consider a form of differences ${ }^{\mathscr{T}} \Delta^{n}$ in order $n \in \mathbb{N}$ to obtain an exact expression of these differences by using condition (38). In the notation of the difference, $\mathscr{T}$ denotes that this difference gives the fractional derivative by transform operator $\mathscr{T}=\mathscr{F}^{-1} \circ \mathscr{F}_{h, \Delta}$ by (37) (see also [8-12]).

At the beginning, we will consider differences ${ }^{\mathscr{T}} \Delta^{n, \pm}$ with even and odd coefficients for simplification.

Definition 9. A general form of differences ${ }^{\mathscr{T}} \Delta^{n, \pm}$ of order $n \epsilon$ $\mathbb{N}$ is defined by the equation

$$
{ }^{\mathscr{T}} \Delta^{n, \pm} f[m]:=\sum_{j=-\infty}^{+\infty} K_{n}^{ \pm}(j) f[m-j],
$$

where the functions $f[m] \in l^{q}(q \geq 2)$ and the kernels $K_{n}^{ \pm}(m) \in l^{p}(p \geq 1)$ are the real-valued functions of discrete variable $m \in \mathbb{Z}$ such that the properties

$$
K_{n}^{ \pm}(-m)= \pm K_{n}^{ \pm}(m)
$$

hold for all $m \in \mathbb{N}$ and $n \in \mathbb{N}$. The operators ${ }^{\mathscr{T}} \Delta^{n, \pm}$ will be called even (“+”) and odd (“-”) $\mathscr{T}$-differences of order $n$.

Remark 10. In the definition we use the sequence space $l^{p}$ $(p>0)$, which is the linear space consisting of all discrete functions (sequences) $f[n]$, where $n \in \mathbb{Z}$, satisfying the inequality

$$
\sum_{n=-\infty}^{+\infty}|f[n]|^{p}<\infty
$$

If $p \geq 1$, then we can define a norm on the $l^{p}$-space by the equation

$$
\|f\|_{p}:=\left(\sum_{n=-\infty}^{+\infty}|f[n]|^{p}\right)^{1 / p} .
$$

The sequence space $l^{p}$ with $p>0$ is a complete metric space with respect to this norm, and therefore it is a Banach space. We can assume that $f[n]$ belongs to the Hilbert space $l^{2}$ of square-summable sequences to apply the Fourier series transform. It is known that if $1 \leq p<q$, then $l^{q} \subset l^{p}$. Therefore, $l^{q} \subset l^{2}$ if $q>2$, and we will consider $f[m] \in l^{q}$ with $q \geq 2$.

Proposition 11. Difference (39), which are defined by convolutions of $K_{n}^{ \pm}(m) \in l^{p}(p>1)$ and $f[m] \in l^{q}(q \geq 2)$ are operators $l^{q} \rightarrow l^{r}$ that map the discrete function $f[m] \in$ $l^{q}(q \geq 2)$ into functions $g^{ \pm}[m] \in l^{r}(r \geq 2)$ such that

$$
g^{ \pm}[m]:={ }^{\mathscr{T}} \Delta^{n, \pm} f[m] \in l^{r},
$$

where $m \in \mathbb{Z}$, and

$$
\frac{1}{r}+1=\frac{1}{p}+\frac{1}{q} .
$$

Proof. It is known that if $f[m] \in l^{q}$ and $K[m] \in l^{p}$, then the inequality

$$
\|K * f\|_{r} \leq\|K\|_{p}\|f\|_{q}
$$

holds, where $r$ is defined by (44) and the star $*$ denotes the convolution. This is Young's inequality for convolutions (see $[13,14]$ and Theorem 276 of [15]). Using Young's inequality in the form

$$
\left\|^{\mathscr{T}} \Delta^{n, \pm} f[m]\right\|_{r}=\left\|K_{n}^{ \pm} * f\right\|_{r} \leq\left\|K_{n}^{ \pm}\right\|_{p}\|f\|_{q},
$$

we get

$$
g^{ \pm}[m]:={ }^{\mathscr{T}} \Delta^{n, \pm} f[m] \in l^{r}
$$

if condition (44) holds and $f[m] \in l^{q}(q \geq 2)$ and $K_{n}^{ \pm}(m) \in l^{p}$ $(p>1)$.

Remark 12. The Fourier series transforms of difference (39) have the form

$$
\begin{aligned}
\mathscr{F}_{h, \Delta}\left\{{ }^{\mathscr{T}} \Delta^{n, \pm} f[m]\right\} & :=\sum_{m=-\infty}^{+\infty} e^{-i k m \mathscr{T}} \Delta^{n, \pm} f[m] \\
& =\widehat{K}_{n}^{ \pm}(k h) \widehat{f}(k) .
\end{aligned}
$$

In order to get (38), we should use

$$
\begin{aligned}
& \widehat{K}_{n}^{+}(k)=|k|^{n}, \\
& \widehat{K}_{n}^{-}(k)=i \operatorname{sgn}(k)|k|^{n} .
\end{aligned}
$$

Using property (40), the kernels $K_{n}^{ \pm}(m)$ can be defined by the equations

$$
\begin{aligned}
& K_{n}^{+}(m)=\frac{1}{\pi} \int_{0}^{\pi} \widehat{K}_{n}^{+}(k) \cos (k m) d k, \\
& K_{n}^{-}(m)=\frac{i}{\pi} \int_{0}^{\pi} \widehat{K}_{n}^{-}(k) \sin (k m) d k .
\end{aligned}
$$


These equations allow us to get exact expressions for the kernels $K_{n}^{ \pm}(m)$.

\section{Exact Expression of Infinite $\mathscr{T}$-Differences}

The exact expressions for the kernels $K_{m}^{ \pm}(n)$, which satisfy conditions (49), are given by the following proposition.

Proposition 13. The kernels $K_{m}^{ \pm}(n)$ of even and odd $\mathscr{T}$-difference (39) for order $m \in \mathbb{N}$, which satisfy conditions (49), are given by the equations

$$
\begin{aligned}
K_{m}^{+}(n)= & \sum_{k=0}^{[(m-1) / 2]} \frac{(-1)^{n+k} m ! \pi^{m-2 k-2}}{(m-2 k-1) !} \frac{1}{n^{2 k+2}} \quad(n \in \mathbb{N}), \\
& +\frac{(-1)^{[(m+1) / 2]} m !(2[(m+1) / 2]-m)}{\pi n^{m+1}} \\
K_{m}^{-}(n)= & -\sum_{k=0}^{[m / 2]} \frac{(-1)^{n+k+1} m ! \pi^{m-2 k-1}}{(m-2 k) !} \frac{1}{n^{2 k+1}} \\
& -\frac{(-1)^{[m / 2]} m !(2[m / 2]-m+1)}{\pi n^{m+1}} \\
K_{m}^{ \pm}(-n)= & \pm K_{m}^{ \pm}(n) \quad(n \in \mathbb{N}), \\
K_{m}^{+}(0)= & \frac{\pi^{m}}{m+1}, \quad(n \in \mathbb{N}), \\
K_{m}^{-}(0)= & 0 .
\end{aligned}
$$

Proof. Substitution of (49) into (50) gives

$$
\begin{aligned}
& K_{n}^{+}(m)=\frac{1}{\pi} \int_{0}^{\pi} k^{n} \cos (k m) d k, \\
& K_{n}^{-}(m)=-\frac{1}{\pi} \int_{0}^{\pi} k^{n} \sin (k m) d k .
\end{aligned}
$$

Then we use equation 2.5.3.5 of [16] that has the form

$$
\begin{aligned}
\int_{0}^{\pi} x^{m} \cos (n x) d x & \\
= & \frac{(-1)^{n+2}}{n^{m+1}} \sum_{k=0}^{[(m-1) / 2]} \frac{(-1)^{k} m !}{(m-2 k-1) !}(\pi n)^{m-2 k-1} \\
& +\frac{(-1)^{[(m+1) / 2]} m !}{n^{m+1}}\left(2\left[\frac{(m+1)}{2}\right]-m\right), \\
\int_{0}^{\pi} x^{m} \sin (n x) d x & (n \in \mathbb{N}), \\
= & \frac{(-1)^{n+1}}{n^{m+1}} \sum_{k=0}^{[m / 2]} \frac{(-1)^{k} m !}{(m-2 k) !}(\pi n)^{m-2 k} \\
& +\frac{(-1)^{[m / 2]} m !}{n^{m+1}}\left(2\left[\frac{m}{2}\right]-m+1\right), \quad(n \in \mathbb{N}),
\end{aligned}
$$

where $[x]$ is the integer part of the value $x$ and $n \in \mathbb{N}$. Here $2[(m+1) / 2]-m=1$ for odd $m$ and $2[(m+1) / 2]-m=0$ for even $m$. As a result, we get (51), (52), and (53).

Examples. The kernels (51) with $m=2$ and $m=4$ have the form

$$
\begin{aligned}
& K_{2}^{+}(n)=\frac{2(-1)^{n}}{n^{2}} \quad(n \neq 0, n \in \mathbb{Z}), \\
& K_{2}^{+}(0)=\frac{\pi^{2}}{3}, \\
& K_{4}^{+}(n)=\frac{4 \pi^{2}(-1)^{n}}{n^{2}}-\frac{24(-1)^{n}}{n^{4}} \quad(n \neq 0, n \in \mathbb{Z}), \\
& K_{4}^{+}(0)=\frac{\pi^{4}}{5} .
\end{aligned}
$$

Using (52) for $m=1$ and $m=3$, we get the examples

$$
\begin{aligned}
& K_{1}^{-}(n)=\frac{(-1)^{n}}{n} \quad(n \neq 0, n \in \mathbb{Z}), \\
& K_{1}^{-}(0)=0, \\
& K_{3}^{-}(n)=\frac{(-1)^{n} \pi^{2}}{n}-\frac{6(-1)^{n}}{n^{3}} \quad(n \neq 0, n \in \mathbb{Z}), \\
& K_{3}^{-}(0)=0 .
\end{aligned}
$$

Let us define $\mathscr{T}$-difference ${ }^{\mathscr{T}} \Delta^{n}$ of order $n \in \mathbb{N}$, that is, a generalization of ${ }^{\mathscr{T}} \Delta^{n, \pm}$ such that conditions (37) and (38) hold.

Definition 14. A general form of $\mathscr{T}$-difference ${ }^{\mathscr{T}} \Delta^{n}$ of order $n \in \mathbb{N}$ is defined by the equation

$$
{ }^{\mathscr{T}} \Delta^{n} f[m]:=\sum_{j=-\infty}^{+\infty} K_{n}(j) f[m-j],
$$

where the function $f[m] \in l^{q}(q \geq 2)$ and the kernel $K_{n}(m) \in$ $l^{p}(p \geq 1)$ are real-valued function of integer variable $m \in \mathbb{Z}$, and

$$
K_{n}(m)=\cos \left(\frac{\pi n}{2}\right) K_{n}^{+}(m)+\sin \left(\frac{\pi n}{2}\right) K_{n}^{-}(m) .
$$

Remark 15. Substitution of (54) into (59) gives

$$
\begin{aligned}
& K_{n}(m) \\
& =\frac{1}{\pi} \int_{0}^{\pi} k^{n}\left(\cos \frac{\pi n}{2} \cos (k m)-\sin \frac{\pi n}{2} \sin (k m)\right) d k
\end{aligned}
$$

As a result, we get

$$
K_{n}(m)=\frac{1}{\pi} \int_{0}^{\pi} k^{n} \cos \left(k m+\frac{\pi n}{2}\right) d k
$$


Remark 16. Using (59), we can represent the $\mathscr{T}$-difference through the even and odd differences in the form

$$
\begin{aligned}
{ }^{\mathscr{T}} \Delta^{n} f[m]= & \cos \left(\frac{\pi n}{2}\right)^{\mathscr{T}} \Delta^{n,+} f[m] \\
& +\sin \left(\frac{\pi n}{2}\right)^{\mathscr{T}} \Delta^{n,-} f[m] .
\end{aligned}
$$

Examples. Let us give exact equations for $\mathscr{T}$-differences of orders $n=1,2,3,4$. The $\mathscr{T}$-difference of first order has the form

$$
{ }^{T} \Delta^{1} f[n]:=\sum_{\substack{m=-\infty \\ m \neq 0}}^{+\infty} \frac{(-1)^{m}}{m} f[n-m] .
$$

The $\mathscr{T}$-difference of second order has the form

$$
{ }^{T} \Delta^{2} f[n]:=-\sum_{\substack{m=-\infty \\ m \neq 0}}^{+\infty} \frac{2(-1)^{m}}{m^{2}} f[n-m]-\frac{\pi^{2}}{3} f[n] .
$$

The $\mathscr{T}$-difference of third order

$$
\begin{aligned}
{ }^{\mathcal{T}} \Delta^{3} f[n] & :=-\sum_{\substack{m=-\infty \\
m \neq 0}}^{+\infty}\left(\frac{(-1)^{m} \pi^{2}}{m}-\frac{6(-1)^{m}}{m^{3}}\right) f[n-m] .
\end{aligned}
$$

The $\mathscr{T}$-difference of fourth order

$$
\begin{aligned}
{ }^{\mathscr{T}} \Delta^{4} f[n] & :=\sum_{\substack{m=-\infty \\
m \neq 0}}^{+\infty}\left(\frac{4 \pi^{2}(-1)^{m}}{m^{2}}-\frac{24(-1)^{m}}{m^{4}}\right) f[n-m] \\
& +\frac{\pi^{4}}{5} f[n] .
\end{aligned}
$$

The minus is used in (64), (66) and the plus is used in (63), (66), since we take into the values $\cos (\pi n / 2)$ and $\sin (\pi n / 2)$ for the order $n \in\{1,2,3,4\}$.

Remark 17. Note that the numerical series of (63)-(66) converge for $f[m] \in l^{q}(q \geq 2)$ since the kernels $K_{n}(m) \in l^{p}$ $(p>1)$ (see Proposition 11). Note that using equation 5.1.2.3 of [16], we can get

$$
\begin{aligned}
\sum_{m=1}^{\infty} \frac{(-1)^{m}}{m^{s}} & =\left(2^{1-s}-1\right) \zeta(s)=-\frac{1}{\Gamma(s)} \int_{0}^{\infty} \frac{x^{s-1}}{e^{x}+1} d x \\
& =T_{s},
\end{aligned}
$$

where $\zeta(s)$ is the Riemann zeta function, $\Gamma(s)$ is the Gamma function, and

$$
\begin{aligned}
& T_{1}=-\ln (2), \\
& T_{2}=-\frac{\pi^{2}}{12}, \\
& T_{3}=-0,90154268 \ldots, \\
& T_{4}=-\frac{7 \pi^{4}}{720} .
\end{aligned}
$$

As a result, series (63)-(66) with $f[m]=1$ for all $m \in \mathbb{Z}$ converge.

Remark 18. Let us note that we can consider the $\mathscr{T}$-difference for $n=-1$. In this case, we have

$$
{ }^{\mathscr{T}} \Delta^{n}:=\sin \left(-\frac{\pi}{2}\right)^{\mathscr{T}} \Delta^{-1,-}=-{ }^{\mathscr{T}} \Delta^{-1,-},
$$

where

$$
\begin{aligned}
K_{-1}(m) & =-K_{-1}^{-}(m)=+\frac{1}{\pi} \int_{0}^{\pi} k^{-1} \sin (m k) d k \\
& =\frac{1}{\pi} \operatorname{Si}(\pi m),
\end{aligned}
$$

and $\operatorname{Si}(z)$ is the sine integral. As a result, we have the difference of first negative order in the form

$$
{ }^{T} \Delta^{-1} f[n]:=\sum_{\substack{m=-\infty \\ m \neq 0}}^{+\infty} \pi^{-1} \operatorname{Si}(\pi m) f[n-m] .
$$

The Fourier series transform $\mathscr{F}_{h, \Delta}$ of this difference is

$$
\mathscr{F}_{h, \Delta}\left({ }^{\mathscr{T}} \Delta^{-1}\right)=(i k h)^{-1} .
$$

This difference corresponds to the integration. Note that a possibility to use the infinite differences for negative orders is not unique. For example, the Grünwald-Letnikov derivatives can be used for orders $\alpha<0$ (see Section 20 in [5] and Section 2.2 in [6]) if the functions $f(x)$ satisfy the condition

$$
|f(x)|<c(1+|x|)^{-\mu}
$$

where $\mu>|\alpha|$.

\section{Properties of Infinite $\mathscr{T}$-Differences}

Let us give examples, which allows us to demonstrate that the $\mathscr{T}$-difference ${ }^{\mathscr{T}} \Delta^{1}$ action is similar to derivative, when applied to polynomials and analytic functions.

Proposition 19. The $\mathscr{T}$-differences ${ }^{\mathcal{T}} \Delta^{1}$ of first order of the power-law function, the sine and cosine functions, and the exponential function have the form

$$
\begin{gathered}
\mathscr{T}^{1} n^{m}=m n^{m-1} \quad(m \in \mathbb{N}), \\
{ }^{T} \Delta^{1} \sin (k n)=k \cos (k n) \quad(k \in \mathbb{R}), \\
{ }^{\mathscr{T}} \Delta^{1} \cos (k n)=-k \sin (k n) \quad(k \in \mathbb{R}), \\
{ }^{T} \Delta^{1} \exp (k n)=k \exp (k n) \quad(k \in \mathbb{R}),
\end{gathered}
$$

where $n \in \mathbb{N}$. 
Proof. The proof is realized by direct calculation of series (63), where we use the Cesaro summations and Poisson-Abel summation (see Section 449 of [17]) for (74) in the form

$$
\begin{gathered}
\sum_{m=1}^{+\infty}(-1)^{m}=-\frac{1}{2}, \\
\sum_{m=1}^{+\infty}(-1)^{m} m^{2 j}=0 \quad(j \in \mathbb{N})
\end{gathered}
$$

and the usual formulas for the product of trigonometric functions and equation 5.4.2.6 of [16] in the form

$$
\sum_{m=1}^{\infty} \frac{(-1)^{m}}{m} \sin (k m)=-\frac{k}{2}
$$

The connection of $\mathscr{T}$-difference ${ }^{\mathscr{T}} \Delta^{n}$ with the even and odd differences ${ }^{\mathscr{T}} \Delta^{n, \pm}$ is given by the following proposition.

Proposition 20. The $\mathscr{T}$-difference ${ }^{\mathscr{T}} \Delta^{n}$ of order $n \in \mathbb{N}$ gives the even and odd $\mathscr{T}$-differences ${ }^{\mathscr{T}} \Delta^{n, \pm}$ by the equations

$$
\begin{aligned}
{ }^{\mathscr{T}} \Delta^{2 m} & =(-1)^{m \mathscr{T}} \Delta^{2 m,+}, \\
\mathscr{T}^{2 m+1} & =(-1)^{m \mathcal{T}} \Delta^{2 m+1,-},
\end{aligned}
$$

where $m \in \mathbb{N}$.

Proof. For even $n=2 m$, where $m \in \mathbb{N}$, the $\mathscr{T}$-difference is given by

$$
\begin{aligned}
{ }^{\mathscr{T}} \Delta^{2 m} & =\cos (\pi m)^{\mathscr{T}} \Delta^{2 m,+}+\sin (\pi m)^{\mathscr{T}} \Delta^{2 m,-} \\
& =(-1)^{m \mathscr{T}} \Delta^{2 m,+} .
\end{aligned}
$$

For odd $n=2 m+1$, where $m \in \mathbb{N}$, the $\mathscr{T}$-difference is

$$
\begin{aligned}
{ }^{\mathscr{T}} \Delta^{2 m+1}= & \cos \left(\pi m+\frac{\pi}{2}\right)^{\mathscr{T}} \Delta^{2 m+1,+} \\
& +\sin \left(\pi m+\frac{\pi}{2}\right)^{\mathscr{T}} \Delta^{2 m+1,-} f[m] \\
= & \cos (\pi m)^{\mathscr{T}} \Delta^{2 m+1,-}=(-1)^{m \mathscr{T}} \Delta^{2 m+1,-} .
\end{aligned}
$$

As a result, we proved (80).

The $\mathscr{T}$-difference of order $n \in \mathbb{N}$ has the following Fourier transform.

Proposition 21. The Fourier series transform $\mathscr{F}_{h, \Delta}$ of the $\mathscr{T}$ difference has the form

$$
\mathscr{F}_{h, \Delta}\left({ }^{\mathscr{T}} \Delta^{n} f[m]\right)(k)=|k h|^{n} \widehat{f}(k)=(i k h)^{n} \widehat{f}(k),
$$

where $\operatorname{sgn}(k)$ is the sign of the variable $k$.
Proof. Using (62) and Euler's formula, we get

$$
\begin{aligned}
\mathscr{F}_{h, \Delta}\left({ }^{\mathscr{T}} \Delta^{n} f[m]\right)(k) \\
=\cos \left(\frac{\pi n}{2}\right) \mathscr{F}_{h, \Delta}\left({ }^{\mathscr{T}} \Delta^{n,+} f[m]\right)(k) \\
+\sin \left(\frac{\pi n}{2}\right) \mathscr{F}_{h, \Delta}\left({ }^{\mathscr{T}} \Delta^{n} f[m]\right)(k) \\
=\left(\cos \left(\frac{\pi n}{2}\right) \widehat{K}_{n}^{+}(k h)\right. \\
\left.+i \operatorname{sgn}(k) \sin \left(\frac{\pi n}{2}\right) \widehat{K}_{n}^{-}(k h)^{n}\right)\left(\mathscr{F}_{h, \Delta} f\right)(k) \\
=\left(\cos \left(\frac{\pi n}{2}\right)|k h|^{n}+i \operatorname{sgn}(k) \sin \left(\frac{\pi n}{2}\right)|k h|^{n}\right) \\
\cdot(\mathscr{F} h, \Delta)(k)=e^{i \pi n \operatorname{sgn}(k h) / 2}|k h|^{n} \widehat{f}(k) \\
=\left(e^{i \pi \operatorname{sgn}(k h) / 2}\right)^{n}|k h|^{n} \widehat{f}(k)=(i \operatorname{sgn}(k h))^{n}|k h|^{n} \\
\cdot \widehat{f}(k)=(i k h)^{n} \widehat{f}(k) .
\end{aligned}
$$

Remark 22. The main property of the $\mathscr{T}$-differences is the Fourier series transform in the form

$$
\mathscr{F}_{h, \Delta}\left({ }^{\mathscr{T}} \Delta^{n} f[m]\right)=(i k h)^{n} \tilde{f}(k) .
$$

As a result, we can consider the inverse Fourier integral transform $\mathscr{F}^{-1}$ of (85) to get

$$
\mathscr{F}^{-1}\left(\mathscr{F}_{h, \Delta}\left({ }^{\mathscr{T}} \Delta^{n}\right)\right)=\mathscr{F}^{-1}\left((i k h)^{n}\right)=h^{n} \frac{\partial^{n}}{\partial x^{n}} .
$$

Let us prove that the $\mathscr{T}$-differences ${ }^{\mathscr{T}} \Delta^{n}$ of integer orders $n \in \mathbb{N}$ are directly related with the derivatives $\partial^{n} / \partial x^{n}$ of orders $n$.

Proposition 23. The transform operators $\mathscr{F}^{-1} \circ \mathscr{F}_{h, \Delta}$ map the $\mathscr{T}$-differences ${ }^{\mathscr{T}} \Delta^{n}$ of integer orders $n \in \mathbb{N}$ into the derivative of orders $n$ :

$$
\mathscr{F}^{-1} \circ \mathscr{F}_{h, \Delta}\left({ }^{\mathscr{T}} \Delta^{n}\right)=h^{n} \frac{\partial^{n}}{\partial x^{n}} .
$$

Proof. Using the Fourier series transform (83) of $\mathscr{T}$-difference, we get

$$
\mathscr{F}^{-1} \circ \mathscr{F}_{h, \Delta}\left({ }^{\mathscr{T}} \Delta^{n}\right)=\mathscr{F}^{-1}\left((i k h)^{n}\right)=h^{n} \frac{\partial^{n}}{\partial x^{n}} .
$$

As a result, we have that the characteristic property of suggested $\mathscr{T}$-differences is the equality

$$
\mathscr{F}_{h, \Delta}\left({ }^{\mathscr{T}} \Delta^{n}\right)=(i k h)^{n}
$$


that leads us to the corresponding equality

$$
\frac{1}{h^{n}} \mathscr{F}^{-1}\left(\mathscr{F}_{h, \Delta}\left({ }^{\mathscr{T}} \Delta^{n}\right)\right)=\frac{\partial^{n}}{\partial x^{n}},
$$

which means that the $\mathscr{T}$-difference of order $n \in \mathbb{N}$ gives the derivative $\partial^{n} / \partial x^{n}$ exactly. The $\mathscr{T}$-difference of order $n \in \mathbb{N}$ is connected with the derivative $\partial^{n} / \partial x^{n}$ not only asymptotically by the limit $h \rightarrow 0$. In the limit $h \rightarrow 0$, we also get

$$
\lim _{h \rightarrow 0} \frac{\mathscr{F}^{-1}\left(\mathscr{F}_{h, \Delta}\left({ }^{\mathscr{T}} \Delta^{n}\right)\right)}{h^{n}}=\frac{\partial^{n}}{\partial x^{n}} .
$$

Therefore the suggested $\mathscr{T}$-difference can be considered not only as approximation of the derivative $\partial^{n} / \partial x^{n}$, but it can also be considered as an exact discrete analog of derivatives of integer orders.

\section{Conclusion}

In this paper, we propose new type of differences that are directly connected with derivatives of integer order in contrast with usual finite differences that can be considered only as approximation of these derivatives. A main characteristic of the suggested differences is that its Fourier series transforms have the power-law form. In the paper we demonstrate that these differences of integer orders $n \in \mathbb{N}$ are directly connected with the derivatives $\partial^{n} / \partial x^{n}$. This is the main advantage of these differences in contrast with all other differences that can be considered as an approximation of integer order derivatives. It allows us to consider an exact discretization of differential equations. We assume that the suggested differences can be interesting in application since they allow us to reflect characteristic properties of complex continua at the microstructural and nanostructural levels by matching lattice models with long-range interactions.

\section{Conflict of Interests}

The author declares that there is no conflict of interests regarding the publication of this paper.

\section{References}

[1] G. Boole, A Treatise on the Calculus of Finite Differences, Cambridge University Press, Cambridge, UK, 2009.

[2] R. P. Agarwal, Difference Equations and Inequalities: Theory, Methods, and Applications, Marcel Dekker, New York, NY, USA, 2nd edition, 2000.

[3] A. K. Grünwald, "About 'limited' derivations their application," Journal of Applied Mathematics and Physics, vol. 12, pp. 441-480, 1897 (German).

[4] A. V. Letnikov, "Theory of differentiation with arbitrary pointer," Matematicheskii Sbornik, vol. 3, pp. 1-68, 1868 (Russian).

[5] S. G. Samko, A. A. Kilbas, and O. I. Marichev, Fractional Integrals and Derivatives Theory and Applications, Gordon and Breach Science Publishers, New York, NY, USA, 1993.

[6] I. Podlubny, Fractional Differential Equations, Academic Press, San Diego, Calif, USA, 1998.
[7] V. P. Maslov, Operator Methods, section 8, Mir, Moscow, Russia, 1976 (Russian).

[8] V. E. Tarasov, "Continuous limit of discrete systems with longrange interaction," Journal of Physics A, vol. 39, no. 48, pp. 14895-14910, 2006.

[9] V. E. Tarasov, "Map of discrete system into continuous," Journal of Mathematical Physics, vol. 47, no. 9, Article ID 092901, 2006.

[10] V. E. Tarasov, "Fractional dynamics of media with long-range interaction," in Fractional Dynamics: Applications of Fractional Calculus to Dynamics of Particles, Fields and Media, Nonlinear Physical Science, pp. 153-214, Springer, Berlin, Germany, 2010.

[11] V. E. Tarasov, “Toward lattice fractional vector calculus," Journal of Physics A, vol. 47, no. 35, Article ID 355204, 51 pages, 2014.

[12] V. E. Tarasov, "Lattice fractional calculus," Applied Mathematics and Computation, vol. 257, pp. 12-33, 2015.

[13] W. H. Young, "On classes of summable functions and their Fourier series," Proceedings of the Royal Society A, vol. 87, no. 594, pp. 225-229, 1912.

[14] W. H. Young, "On the multiplication of successions of Fourier constants," Proceedings of the Royal Society of London Series A: Containing Papers of a Mathematical and Physical Character, vol. 87, no. 596, pp. 331-339, 1912.

[15] G. H. Hardy, J. E. Littlewood, and D. G. Polya, Inequalities, Cambridge University Press, Cambridge, UK, 2nd edition, 1952.

[16] A. P. Prudnikov, Y. A. Brychkov, and O. I. Marichev, Integrals and Series, Vol. 1: Elementary Functions, Gordon and Breach, New York, NY, USA, 1986.

[17] G. M. Fichtenholz, Differential and Integral Calculus, vol. 2, Nauka, Moscow, Russia, 7th edition, 1969 (Russian). 


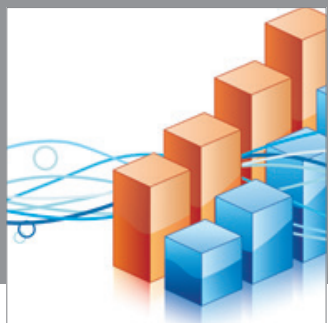

Advances in

Operations Research

mansans

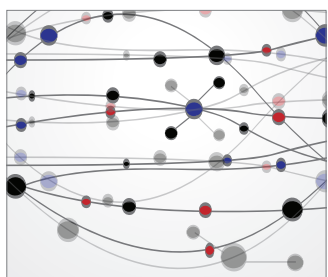

The Scientific World Journal
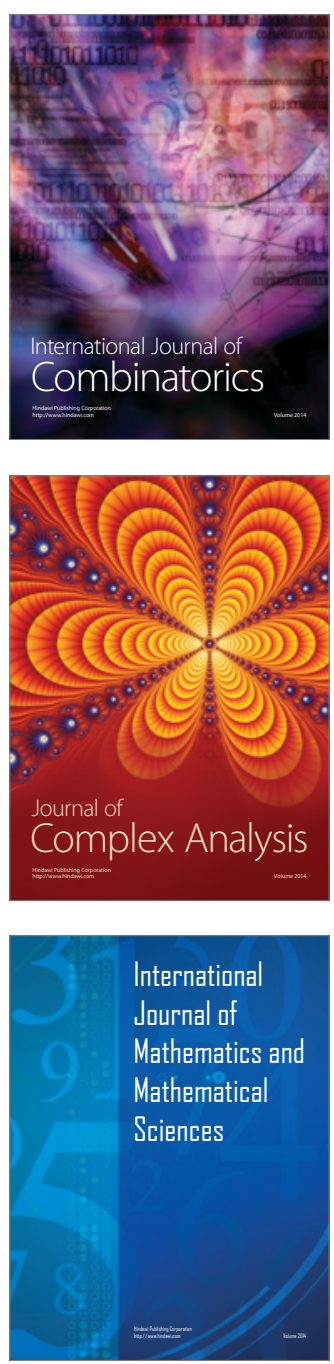
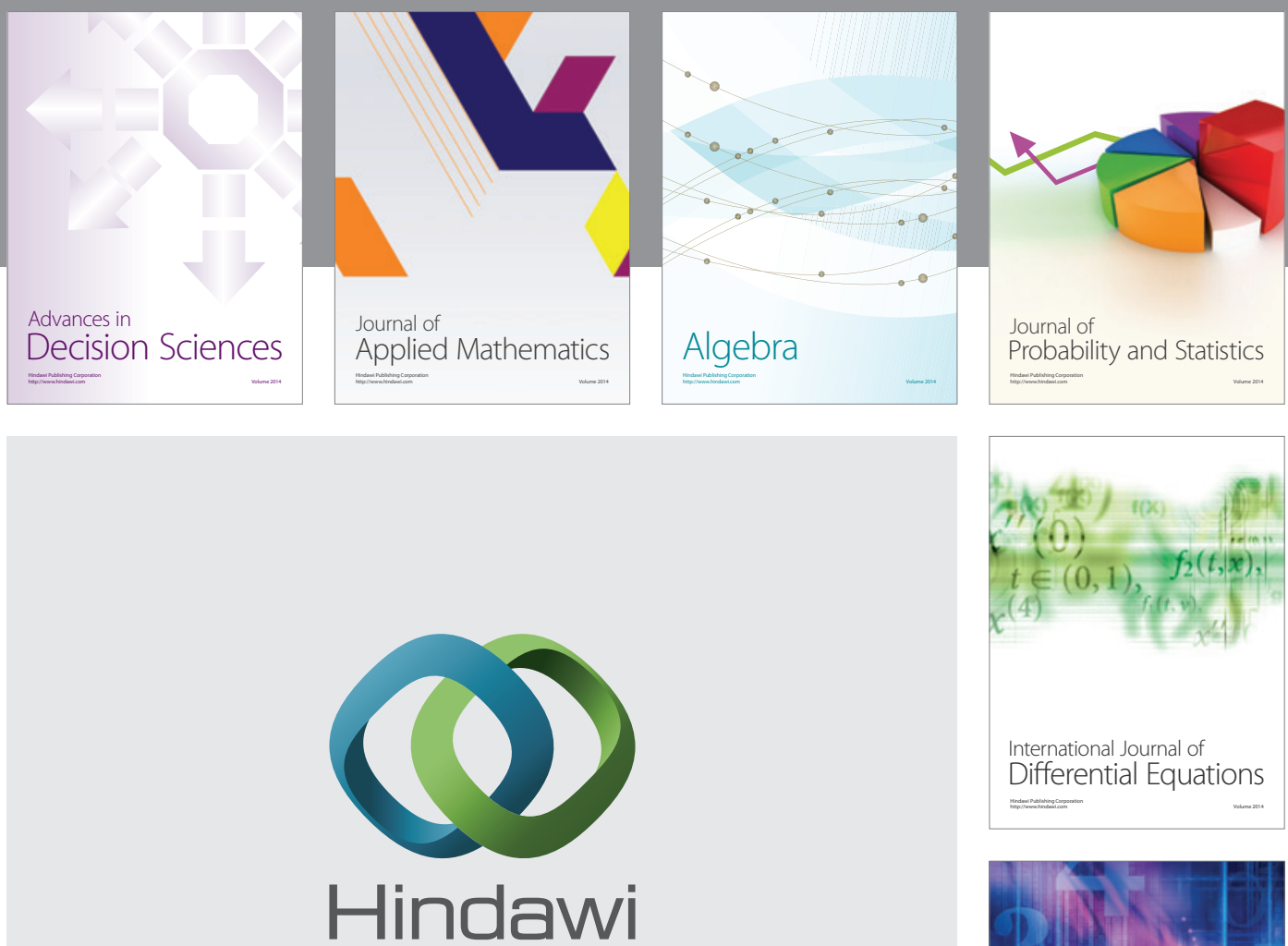

Submit your manuscripts at http://www.hindawi.com
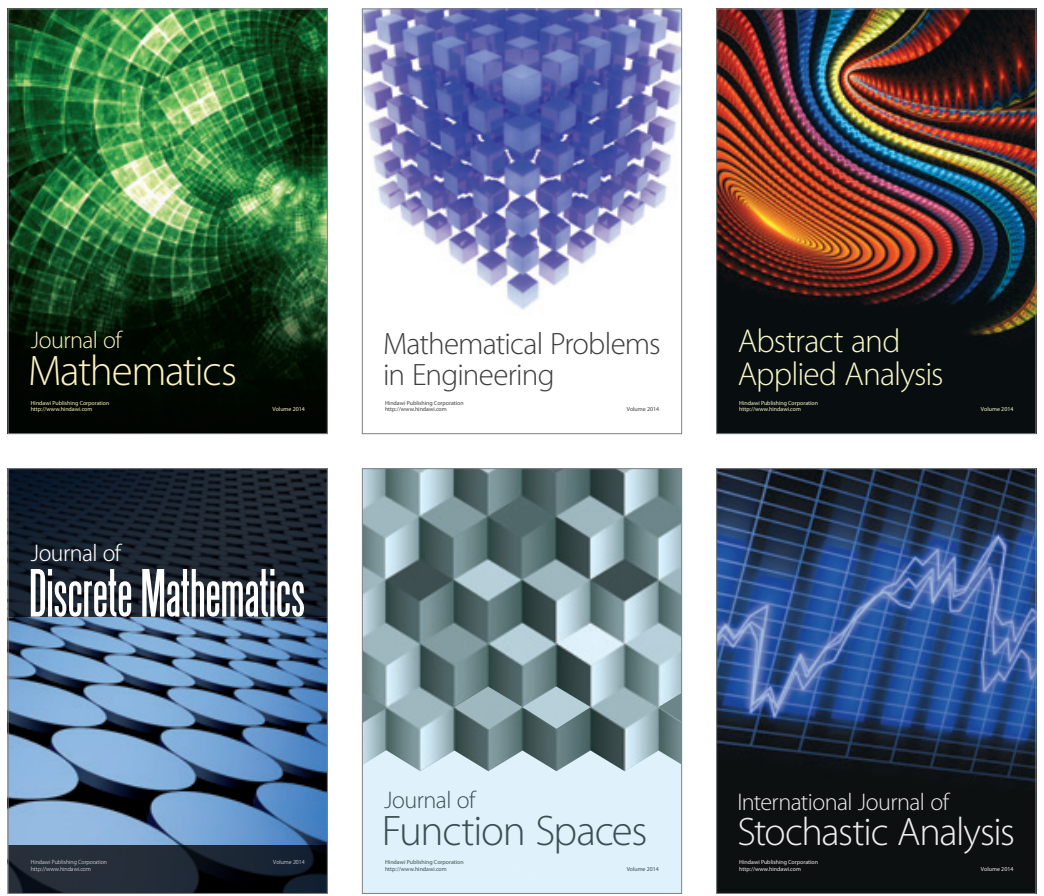

Journal of

Function Spaces

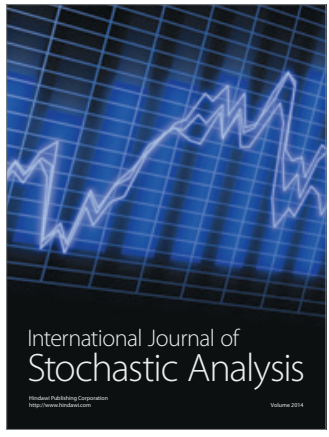

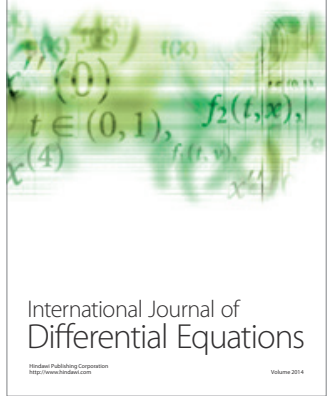
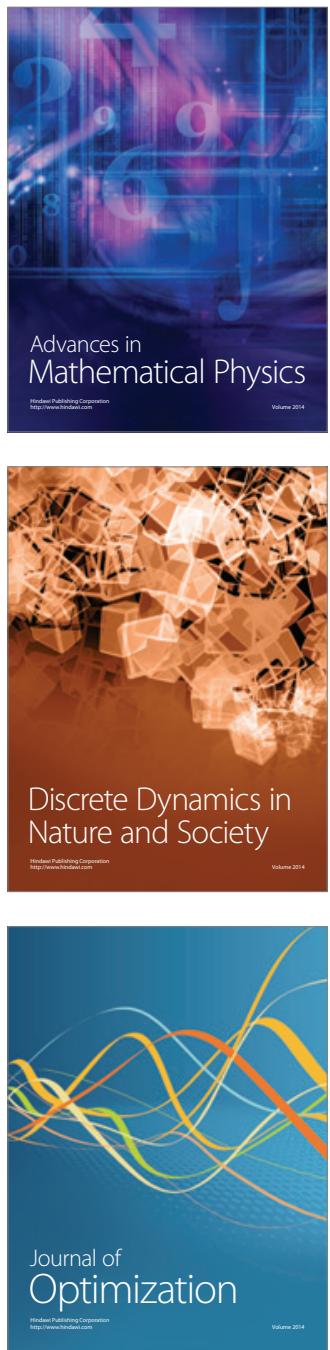